\title{
Five additions to the list of Sepsidae (Diptera) for Vietnam: Perochaeta cuirassa sp. n., Perochaeta lobo sp. n., Sepsis spura sp. n., Sepsis sepsi Ozerov, 2003 and Sepsis monostigma Thompson, 1869
}

\author{
Yuchen Ang ${ }^{\dagger}$, Rudolf Meier ${ }^{\ddagger}$ \\ Evolutionary Biology Laboratory, Department of Biological Sciences, National University of Singapore, Singa- \\ pore 117543, Republic of Singapore \\ † urn:lsid:zoobank.org:author:6DB33151-6995-4462-8AF9-470E5E8784C7 \\ ¥urn:lsid:zoobank.org:author:854F05EB-7323-496B-8F20-AD6ACFECC1EB \\ Corresponding author: Yuchen Ang (yuchen@nus.edu.sg) \\ Academiceditor:Martin Hauser $\mid$ Received 4September 2010|Accepted 15November 2010|Published 29November 2010 \\ urn:lsid:zoobank.org:pub:327B3E99-46C3-4D1E-9B74-5C3369F8969F \\ Citation: Ang Y, Meier R (2010) Five additions to the list of Sepsidae (Diptera) for Vietnam: Perochaeta cuirassa sp. n., \\ Perochaeta lobo sp. n., Sepsis spura sp. n., Sepsis sepsi Ozerov, 2003 and Sepsis monostigma Thompson, 1869. ZooKeys 70: \\ 41-56. doi: 10.3897/zookeys.70.766
}

\begin{abstract}
A recent collecting trip to Vietnam yielded three new species and two new records of Sepsidae (Diptera) for the country. Here we describe two new species in the species-poor genus Perochaeta (Perochaeta cuirassa sp. n. and P. lobo sp. n.) and one to the largest sepsid genus Sepsis (Sepsis spura sp. n.) which is also found in Sumatra and Sulawesi. Two additional Sepsis species are new records for Vietnam (Sepsis sepsi Ozerov, 2003; Sepsis monostigma Thompson, 1869). We conclude with a discussion of the distribution of Perochaeta and the three Sepsis species.
\end{abstract}

\section{Keywords}

Sepsidae, Perochaeta, Sepsis, new species, new records, Vietnam

Copyright Y. Ang, R. Meier. This is an open access article distributed under the terms of the Creative Commons Attribution License, which permits unrestricted use, distribution, and reproduction in any medium, provided the original author and source are credited. 


\section{Introduction}

The Sepsidae are a moderately large, cosmopolitan family of saprophagous flies, with over 300 extant species recorded from all zoogeographic regions (Ozerov 2005). Most species are attracted to dung, carrion, and other malodorous, decaying organic substrates (Pont and Meier 2002); i.e., by using different substrates in different microhabitats, the sepsid fauna from a specific locality can be quickly explored. Separating sepsids from the remaining saprophagous insects is also relatively straightforward because most sepsids can be easily recognized based on the constriction of the first two abdominal segments which gives the flies a wasp- or ant-like habitus.

Here we update an existing species list for Vietnam by adding five species: three are new to science while two others are new records. The relatively large number of additions is due to the fact that the Vietnamese sepsid fauna remains poorly studied (e.g. Ozerov 1993, Iwasa and Thinh 2008). The current species list comprises 21 species in six genera and is based on the sepsid world catalogue (Ozerov 2005) and subsequent taxonomic research by Iwasa and Thinh (2008). We complement this list by adding five species that were collected during a brief collecting trip in July 2010: Perochaeta cuirassa sp. n., P. lobo sp. n., Sepsis spura sp. n., S. sepsi and S. monostigma.

\section{Materials and methods}

All five species were collected between 11-16 July 2010 from Northern Vietnam (Ba Vi National Park and Sa Pa Valley). Cow dung was placed in various habitats for at least a few hours to attract sepsids, which were then caught by sweep-netting. Additional material for Sepsis sepsi and S. spura were also collected previously in Indonesia (Sulawesi and Sumatra) in 2007 and 2009.

Specimens were photographed using a Leica Z16 APO-A stereomicroscope fitted with a DFC425 digital microscope camera, and then digitally traced to illustrations using a Wacom(C) PTZ 630 tablet. We also amplified and sequenced a 544-bp fragment of cytochrome oxidase c subunit I (COI) within the DNA barcoding region for the two new Perochaeta species based on the methods described in Tan et al. (2010). All type specimens and additional material are vouchered in 95\% ethanol and kept in the Cryogenic Collection of the Raffles Museum of Biodiversity and Research (RMBR), National University of Singapore, Singapore. We adopt the taxonomic terminology as described by Merz and Haenni (2000) for adult morphology (excluding terminalia) and Sinclair (2000) for male genitalia.

\section{Taxonomy}

Describing new species in genera that have not been revised recently requires extra care and justification, because the risk of creating new synonyms based on overlooked or misinterpreted species in the literature is high. Fortunately, this is not the case for Pero- 
chaeta, which has only three described species (see Ozerov 2005 and Ang et al. 2008) and no synonyms. In addition, the descriptions and illustrations for the described species are of good quality. Furthermore, molecular data are consistent with distinct species: the two new Perochaeta species are separated by 3.3\% for the barcoding gene COI (uncorrected pairwise distances); while the distances of either to Perochaeta dikowi are $11.4 \%$ and $11.8 \%$ (see Table 1 for variable base pairs).

Describing a new Oriental Sepsis species in the absence of a generic revision is more problematic given that the genus is the largest in Sepsidae (ca. 80 described, valid species). Of the 23 Sepsis recorded in the Oriental region (Ozerov 2005), the Sepsis species described here closely resembles the widespread $S$. nitens Wiedemann, 1824 which has two synonyms (S. brevicosta Brunetti, 1910 and S. tuberculata Duda, 1926). Duda's (1926) description and fore leg illustration of $S$. tuberculata are sufficiently detailed to confirm that it is indeed a synonym of $S$. nitens. However, Brunetti's (1910) rather vague description of $S$. brevicosta based on one male from Calcutta and a few females from localities in other Indian localities (Calcutta, Shencotta, Tinpahar and Pusa), is more difficult to interpret. He describes $S$. brevicosta's fore femur as having "a small bump [on the ventromedial region] with three or four strong short spines". This description of a "bump" is in agreement with $S$. nitens (Fig. 21) while the ventromedial protrusion of the new species of Sepsis is shaped more like a spur (Figs 24, 26). In addition, Brunetti's S. brevicosta is known from India, while the new Sepsis species described here is only known from Vietnam (Lào Cai) and Indonesia (Sumatra and Sulawesi).

As argued elsewhere, new species are hypotheses that are dependent on species concepts; it is therefore desirable that authors who describe species are explicit about which species concept was used and whether other species concepts would come to different conclusions (Laamanen et al. 2003, Tan et al. 2008, 2010). Here we apply the Hennigian species concept (Meier and Willmann 2000) and use morphology and DNA sequence data (for Perochaeta) to estimate the species boundaries. The two new Perochaeta species are sympatric and the new Sepsis species is parapatric with $S$. nitens. In both cases we have not seen any intermediate specimens so that there is no evidence for hybridization. This supports our species hypotheses. However, both Perochaeta species are so rare that this test is relatively weak. As pointed out by Lim

Table I. Seventy-two variable base pairs in a 544-bp COI sequence fragment for three Perochaeta (Positions according to Drosophila melanogaster COI).

\begin{tabular}{l|l}
\hline Base Pair No. & $\begin{array}{l}00000000000011111111112222222222222333333333333333333344444444444555555555 \\
455666778899223334457800223444556770000112222367888991122244599011245677 \\
615036251713362384701614251367281094679254578668147061736724325709895779\end{array}$ \\
\hline $\begin{array}{l}\text { Perochaeta } \\
\text { dikowi }\end{array}$ & TCTTATTAGTNGTATCTCCAAAAAAGAACCAAAATCTTATTATACTTTTACTTATTCTTATCTAAACATTCT \\
\hline $\begin{array}{l}\text { Perochaeta } \\
\text { cuirassa }\end{array}$ & CCACTAAGCATACTATCTTGTTGTGATATTAACGATACTCCTCTTCTGCAGATTAATCCTATAAATTTAATA \\
\hline $\begin{array}{l}\text { Perochaeta } \\
\text { lobo }\end{array}$ & CTACTAAACATATTATCTTGTTATGATCTTGGTGATACTTTTCTTCCACGGACTAATCCTACAGCTTTAACA \\
\hline
\end{tabular}


et al. (in press), in such cases descriptions should only be prepared if the species are unusually distinct. This is the case here, whereby the two new species can be clearly distinguished based on both morphology [cf. sternites and hypopygia of $P$. cuirassa (Figs 1-4) and P. lobo (Figs 6-9)] and DNA sequence data (Table 1). When the distributional and morphological data are applied to the remaining species concepts in Wheeler and Meier (2000), most support the same species boundaries. The only exception is Mishler and Theriot's (2000) phylogenetic species concept that requires a phylogenetic analysis before species can be delimited. However, such analyses are currently unavailable. We now describe the new Perochaeta and Sepsis species and state the new records for the two Sepsis species.

\section{Perochaeta cuirassa Ang 2010, sp. n.}

urn:lsid:zoobank.org:act:76BA1CDF-D467-45D0-9898-305C21045C6D

Figs $1-5$

Material. Holotype. ô (RMBR), Vietnam, Lào Cai Province, Sa Pa Valley. Baited with cow dung at forest edge next to a small cascade alongside highway, ca. $850 \mathrm{~m}$ along the road westward of the Thác bạc (Silver Waterfall) tourist attraction [22 $23^{\prime} 23.90^{\prime \prime} \mathrm{N}$ $103^{\circ} 44^{\prime} 50.32$ "E, elevation 2600m above sea level, ASL]. Collected 16.VII.2010 (Ang Y). Paratypes. $2 \circlearrowleft$ (RMBR), collected from same locality and time as holotype.

Etymology. The specific epithet refers to the shape of the main scleral plate for the $4^{\text {th }}$ sternite, which resembles a cuirass or breastplate armor.

Diagnosis. Adult male Perochaeta cuirassa is very similar to Perochaeta lobo and can only be reliably distinguished from the latter based on the $4^{\text {th }}$ sternite [cf. P. cuirassa (Fig. 1) and P. lobo (Fig. 6)]: The sternite in P. cuirassa lacks distinct lobes on the posterior end of the $4^{\text {th }}$ sternite, while the sternite brush is thick and squat (as opposed to long and thin in $P$. lobo), and the main scleral plate is much broader (long as wide) than in P. lobo (twice long as wide). The hypopygium [cf. P. cuirassa (Figs 2-4) and P. lobo (Figs 7-9)] is also distinct, with $P$. cuirassa bearing a large median, decussating protrusion on the dorsal side of the surstylus, while $P$. lobo has a sub-median protrusion on the ventral side of the surstylus. Perochaeta cuirassa is also readily distinguished from all other Perochaeta species based on the morphology of the $4^{\text {th }}$ sternite and hypopygium: The sternites brush of $P$. cuirassa (Fig. 1) has significantly more bristles ( $>40$ per brush) than either $P$. hennigi Ozerov, 1992 (Fig. 10) or P. dikowi (Fig. 12), both of which have only 5-6 large bristles in addition to a few weaker bristles. Perochaeta cuirassa also has strong bristles lining the distal margin of the sternite, which are not found in $P$. dikowi or $P$. hennigi. The surstylus of $P$. cuirassa (Fig. 2) resembles that of $P$. hennigi (Fig. 11), but can be distinguished by the large median surstylus projection, which is long and curved in $P$. cuirassa but short and broadly triangular in $P$. hennigi. Both $P$. dikowi (Fig. 13) and $P$. orientalis (De Meijere, 1913) (Fig. 14) lack large median projections. Perochaeta cuirassa can further be distinguished by the radial-medial cross-vein dividing the discal-medial cell which is in a ratio of $3: 1$ in P. cuirassa, 2.5: 1 in $P$. dikowi, $2: 1$ in $P$. hennigi and $1: 1$ in P. orientalis. 

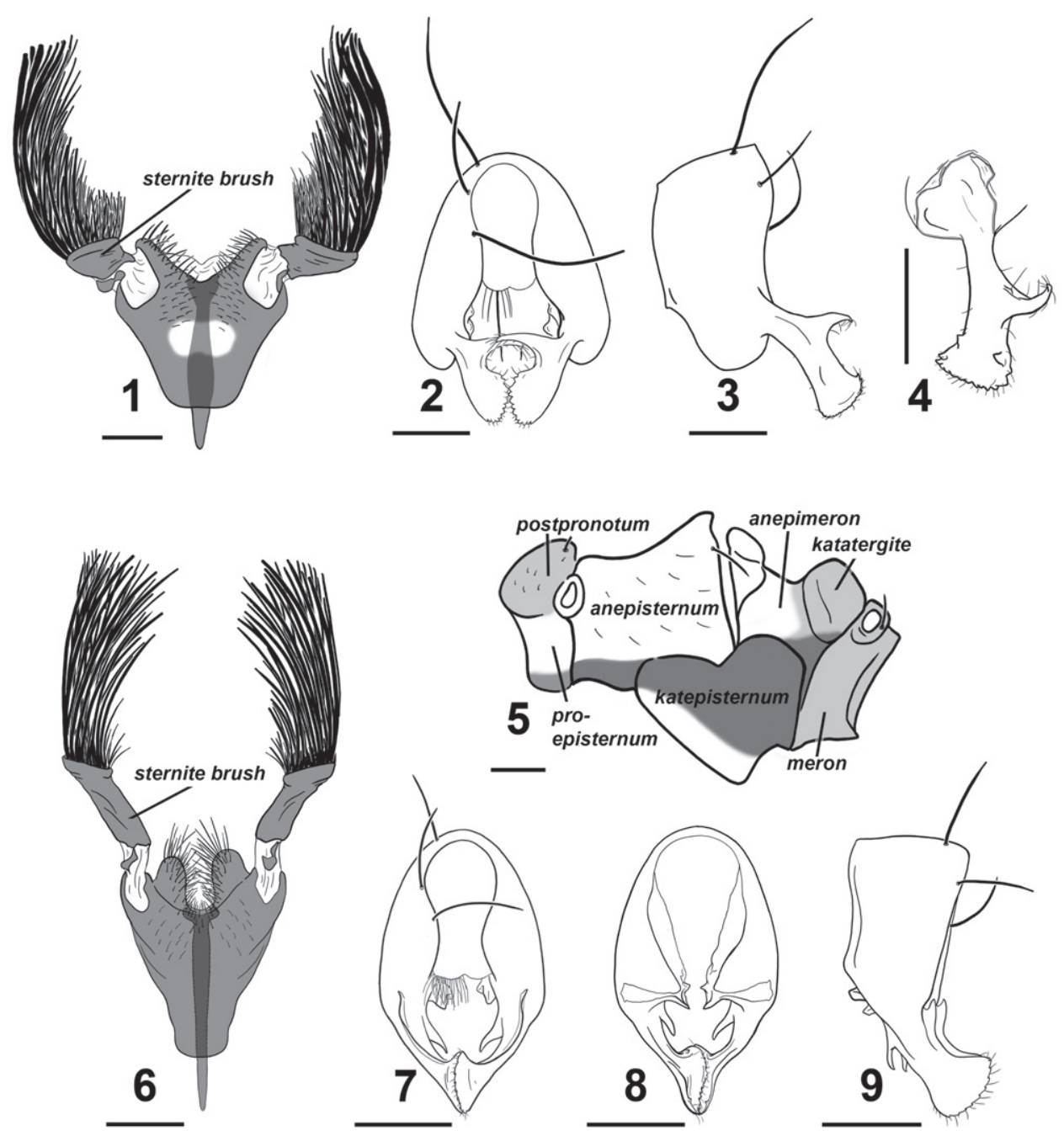

Figures I-9. Perochaeta cuirassa and Perochaeta lobo ô. Perochaeta cuirassa: $\mathbf{1} 4^{\text {th }}$ sternite, ventral $\mathbf{2}$ hypopygium, dorsal $\mathbf{3}$ hypopygium, lateral $\mathbf{4}$ surstylus, lateral (inward facing) $\mathbf{5}$ pleural pruinosity pattern, lateral. Perochaeta lobo: $\mathbf{6} 4^{\text {th }}$ sternite, ventral $\mathbf{7}$ hypopygium, dorsal 8 hypopygium, ventral $\mathbf{9}$ hypopygium, lateral. Scale bars: $0.5 \mathrm{~mm}$.

Description (male). Colour. Head capsule mostly black except for thin yellow strip along subgena and parafacial area. Lunule, facial carina and antennae light brown; antennal groove dark brown. Proboscis brown. Thorax, scutellum and abdomen wholly black. Legs largely yellow except for the following: basal regions of fore coxa brown, mid and rear femora with a dark half-ring subapically (edges of which are diffuse on the apical edge), basal half of mid and rear tibiae dark brown. All tarsi with tarsomeres 3-5 brown; tarsomeres 1-2 yellow with brown region apically. Wing clear except for basicostal cell and basal third of costal cell, which is brown. Veins 
dark brown. Calypter creamy, margin and fringe-hairs yellowish. Haltere milky yellow with brown base.

Head. Roundish, facial carina short and shallow, facial area receding. Gena and parafacial region narrow. Ocellar prominence and occipital region lightly microtomentose. Chaetotaxy: 1 ocellar, 1 divergent postocellar, 1 outer vertical; inner vertical absent. Orbital very reduced to absent. 2 vibrissae. 3-4 postocular. Lower fascial margin lined with setulae.

Thorax. Scutum, postpronotum, scutellum and subscutellum wholly microtomentose. Mediotergite microtomentose but glossy in the medial region. Scutellum twice wide as long. Pleural pruinosity pattern (Fig. 5): Proepisternum glossy with ventral region microtomentose. Anepisternum largely glossy with anterioventral region densely microtomentose. Katepisternum largely with dense tomentosity except for glossy anterioventral region. Anterior side of anepimeron glossy while posterior side lightly microtomentose and posterioventral region densely microtomentose. Postpronotum, katatergite, meron and metepimeron lightly-dusted. Chaetotaxy: 1 apical scutellar, 1 reduced, setulae-like basal scutellar, 1 dorsocentral, 1 postalar, 1 supraalar, 2 notopleural, 1 postpronotal, 1 anepisternal and 1 posterior spiracular. Postpronotoum, prescutum and anepisternum with few, sporadic setulae.

Legs. Forelegs unmodified in males; all femora and tibiae without posteriodorsal or anteriodorsal setae. Mid tibia with row of short setae on anterior apex. Rear tibia with barely-visible osomoterial patch on medial posteriodorsal side. Rear basitarsus with three ventral dark spines basally.

Wings. Without pterostigma. Veins bare. Covered with microtrichiae except for costal, subcostal, basal-medial and posterior-cubital cells, as well as base of cells $\mathrm{r} 1$ and $\mathrm{r} 2+3$. Microtrichiae sparse on basal region of basal-radial and discal-medial cells. Radial-medial cross-vein divides discal-medial cell by ratio of $3: 1$. Length: $4.6-4.9 \mathrm{~mm}$.

Abdomen. Glossy black; syntergite $1+2$ - tergite 5 normal, tergite 6 missing, syntergite $7+8$ present and extending ventrad as a narrow sclerite. Spiracles $1-4$ on intersegmental membrane, spiracle 5 on ventral margin of tergite 5 , spiracle 7 and 8 adjacent on margin of syntergite $7+8$. Sternite 1 broadly rectangular while sternite 2 is triangular, tapering posteriorly; sternite 3 is oblong. $4^{\text {th }}$ sternite heavily modified (Fig. 1); greatly expanded, long as is wide, and in the shape of a breastplate. Two stout moveable appendages (= sternite brushes) branch off laterally; each appendage resembles a painter's brush, with large, thick bristles on the outer side and shorter, thinner bristles on the inner side. Posterior edge of $4^{\text {th }}$ sternite invaginated with strong setae lining the outer discal margin as well as submedially. A Y-shaped apodeme extends underneath and anterior to the $4^{\text {th }}$ sternite.

Hypopygium (Figs 2-4). Cercal plate with two very weak lobes; distal margin of each lobe covered with numerous setae. Hypopygium triangular with a two tooth-like projections on the inner side basal to where the surstylus branches off (Fig. 4). Surstylus itself fused to hypopygium, angled dorsally, and branches off subterminally (Fig. 3). Each surstylus has a large hook-like median projection that curves dorsally and decussates. Terminal section of surstylus shaped like a scapula, with cuticular "teeth" and 
setulae on distal margin, and a small inward-facing "tooth" on both the ventral and dorsal region subterminally pointing towards the median.

Distribution. Vietnam (Lào Cai).

\section{Perochaeta lobo Ang 2010, sp. n.}

urn:lsid:zoobank.org:act:5B7C6CFA-9DCC-4377-9893-CBD789E333E9

Figs 6-9

Material. Holotype. $\overbrace{}^{\lambda}$ (RMBR), Vietnam, Lào Cai Province, Sa Pa Valley. Baited with cow dung at forest edge next to a small cascade alongside highway, ca. $850 \mathrm{~m}$ along the road westward of the Thác bạc (Silver Waterfall) tourist attraction $\left[22^{\circ} 23^{\prime} 23.90 \mathrm{~N}\right.$ $103^{\circ} 44^{\prime} 50.32 \mathrm{E}$, elevation 2600m above sea level, ASL]. Collected 16.VII.2010 (Ang Y).

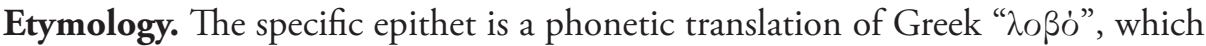
refers to the large, distinct lobe found on each lateral half on the posterior margin of the $4^{\text {th }}$ sternite.

Diagnosis. The adult male is very similar to $P$. cuirass and can only be reliably distinguished based on the $4^{\text {th }}$ sternite and hypopygium. The $4^{\text {th }}$ sternite [cf. P. lobo (Fig. 6) and $P$. cuirassa (Fig. 1)] can be distinguished to species by the presence of distinct lobes on the posterior end, the long, thin sternite brush (as opposed to short and squat in P. cuirassa), and the sternite itself being narrower (half wide as long). The structure of the hypopygium (Figs 7-9) is also diagnostic given that it is the only Perochaeta with a surstylus that has a dorsal flap along its length and a long distal-pointing projection sub-basally. Other diagnostic characters that distinguish $P$. lobo from $P$. dikowi, $P$. hennigi and $P$. orientalis as described in diagnosis for $P$. cuirassa.

Description (male). Colour. As described in P. cuirassa except for fore and rear basitarsi, which are brown with yellow base, mid basitarsus yellow with slight brown region apically.

Head. As described in P. cuirassa.

Thorax. As described in P. cuirassa; pleural pruinosity pattern as in Fig. 5.

Legs. Forelegs unmodified; as described in P. cuirassa.

Wings. Coloration, venation and microtrichia distribution as described in P. cuirassa. Length: $4.2 \mathrm{~mm}$.

Abdomen. Tergites and sternites $1-3$ as described in P. cuirassa. Sternite 4 heavily modified (Fig. 6); almost twice long as wide and raised from the abdomen. Posterior edge of $4^{\text {th }}$ sternite deeply invaginated and raised to form two large distinct lobes densely populated with strong bristles, mainly lining the outer discal margin. Two long, thin moveable appendages (= sternite brushes) branch off laterally on the posterior end of the sclerite, each with large, thick bristles on the outer region and some shorter, thinner bristles closer to the inside. A Y-shaped apodeme extends underneath and anterior to the $4^{\text {th }}$ sternite.

Hypopygium (Figs 7-9). Cercal plate with two very weak lobes; distal margin covered with numerous setae. Hypopygium triangular with bicuspid projection before the 

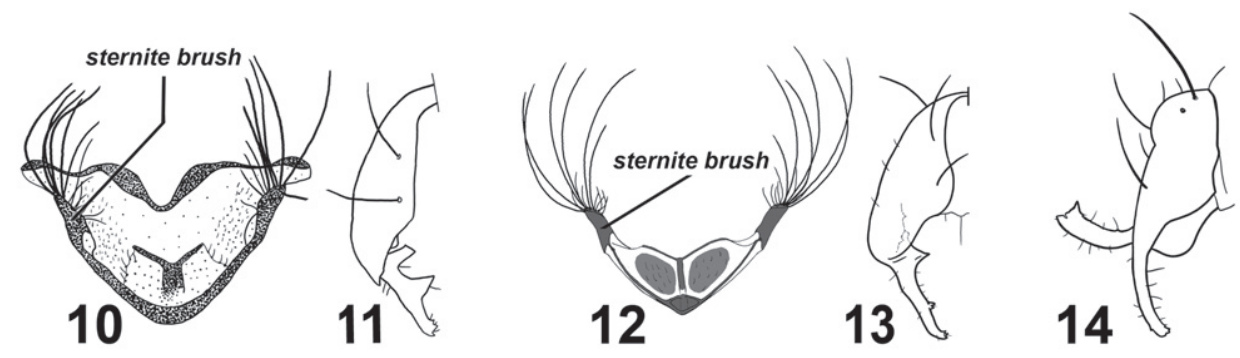

Figures 10-14. Various Perochaeta $4^{\text {th }}$ sternites and hypopygia. Perochaeta hennigi $\widehat{o}$ redrawn from Ozerov (1992): $104^{\text {th }}$ sternite, ventral I I hypopygium (half), dorsal. Perochaeta dikowi $\widehat{\text { ô }}$ redrawn from Ang et al. (2008): $\mathbf{I} 24^{\text {th }}$ sternite, ventral $\mathbf{3}$ hypopygium (half), dorsal. Perochaeta orientalis ô redrawn from Duda (1926): 14 hypopygium, lateral.

base of the surstylus. Surstylus itself fused to hypopygium and branches off terminally, with a dorsal flap along its length and a longish distal-pointing projection sub-basally. Terminal section of surstylus shaped like a scapula, with distal-pointing cuticular "teeth" and setulae on distal margin, and a very small inward-facing "tooth" on both the ventral and dorsal region subterminally pointing towards the median.

Distribution. Vietnam (Lào Cai).

\section{Sepsis monostigma Thompson, 1869}

Figs $15-17$

Sepsis monostigma Thompson, 1869. Kongliga svenska fregatten Eugenies resa omkring Jorden, 2(1): 443.

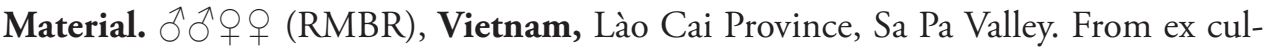
ture established from $q$ collected from dung on cow farm, $4 \mathrm{~km} \mathrm{NE}$ of Sa Pa town [2221'28.19"N 10351'53.35"E, elevation 1250m ASL]. Collected 15.VII.2010 (Ang Y).

Taxonomic remarks. Sepsis monostigma is an Oriental species that resembles Sepsis pseudomonostigma Ursu, 1969 but is geographically exclusive from S. pseudomonostigma (which has only been recorded in South and South-east Europe and Central Asia). Sepsis monostigma can be differentiated by the two long medioventral spines (one short spine in S. pseudomonostigma) on the fore femur (Fig. 15), lack of ventromedial spinules on the fore tibia (Fig. 16) and surstylus (Fig. 17) being much thinner than that in S. pseudomonostimga.

Distribution. China (Guandong), Taiwan, India (Jammu and Kashmir, Uttar Pradesh, West Bengal), Japan (Hokkaido Is., Honshu Is., Kyushu Is.), Korea, Philippines (Luzon Is.), Sri Lanka, South Russia (Amurskaya Oblast', Irkutskaya Oblast', Khabarovskiy Kray, Krasnoyarskiy Kray, Novosibirskaya Oblast', Primorskiy Kray, Sakhalinskaya Oblast'), Vietnam (Lào Cai). 

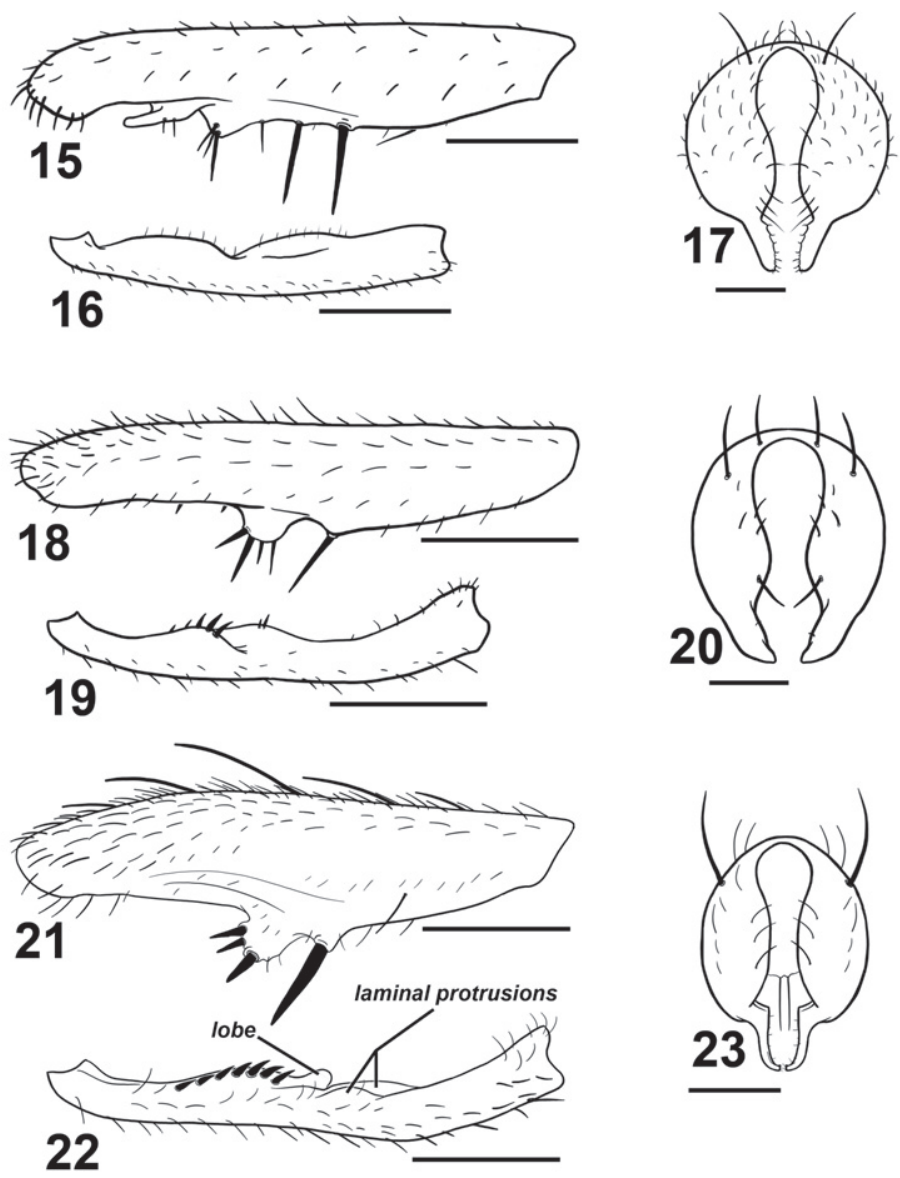

Figures 15-23. Various Sepsis forelegs and hypopygia. Sepsis monostigma: $\mathbf{1 5}$ fore femur, posterior $\mathbf{1 6}$ fore tibia, posterior $\mathbf{1 7}$ hypopygium, dorsal. Sepsis sepsi: $\mathbf{1 8}$ fore femur, posterior $\mathbf{1 9}$ fore tibia, posterior $\mathbf{2 0}$ hypopygium, dorsal. Sepsis nitens: $\mathbf{2}$ I fore femur, posterior $\mathbf{2 2}$ fore tibia, posterior $\mathbf{2} \mathbf{3}$ hypopygium, dorsal. Scale bars: $0.5 \mathrm{~mm}$.

\section{Sepsis sepsi Ozerov, 2003}

Figs $18-20$

Sepsis sepsi Ozerov 2003. Zoologicheskiy zhurnal, 82, 10: 1276.

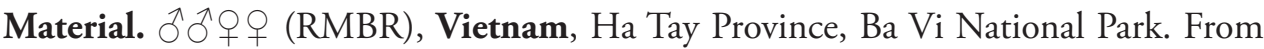
ex culture established from 9 collected from rubbish dump near temple at summit of mountain [ $21^{\circ} 3^{\prime} 45.84^{\prime \prime} \mathrm{N} 105^{\circ} 21^{\prime} 57.63^{\prime \prime E}$, elevation 800m ASL]. Collected 11.VII.2010 (Ang Y). 2 ô (RMBR), Indonesia, West Sumatra, Bukit Tingei Regency, Tanjung Mutiara Dist., Bantar Gadang Beach, [0²4.792"S 9956.307"E 0m ASL]. Collected 6.VII.2007 (Lohman D). 
Taxonomic remarks. Sepsis sepsi bears some resemblance to $S$. nitens and was initially thought to be such by Hennig but later identified it as a 'Sepsis n. sp.' (1941), and was formally described by Ozerov (2003). Sepsis sepsi can be distinguished from $S$. nitens based on the row of four large spines on a large rounded ventromedial bump of the fore femur (Fig. 18) as opposed to three spines arranged triangularly on a slightly proclinate bump in S. nitens (Fig. 21). The fore tibia (Fig. 19) lacks a rounded lobe present in S. nitens (Fig. 22), and the surstylus differs in structure [cf. S. sepsi (Fig. 20) and S. nitens (Fig. 23)].

Distribution. Indonesia (Sumatra, Sumbawa), Vietnam (Ha Tay).

\section{Sepsis spura Ang 2010, sp. n.}

urn:lsid:zoobank.org:act:581DEFE8-8274-4365-BCDF-7F34BB59A258

Figs 24-31

Material. Holotype. $\widehat{\jmath}$ (RMBR), Vietnam, Lào Cai Province, Sa Pa Valley. Collected from dung on cow farm, $4 \mathrm{~km} \mathrm{NE}$ of Sa Pa town [22 $21^{\prime} 28.19^{\prime \prime N ~ 10351 ' 53.35 " E, ~}$ elevation 1250m ASL]. Collected 15.VII.2010 (Ang Y). Paratypes. 2 ô (RMBR), Indonesia, West Sumatra, Bukit Tingei Regency, Tanjung Mutiara Dist., Bantar Gadang Beach, [0²4.792"S 9956.307"E 0m ASL]. Collected 6.VII.2007 (Lohman D). ${ }^{\circ}$ (RMBR), Indonesia, N. Sulawesi, Tondano Province, Kampung Jawa [1 ${ }^{\circ} 17^{\prime} 18.11^{\text {"N, }}$ 124'52'30.05"E, elevation 650m ASL]. Collected 12.V.2009 (Ang Y).

Etymology. The specific epithet old English for "spur", and refers to the distinct spur-like medioventral tubercle found on the male fore femur.

Diagnosis. Adult males of Sepsis spura closely resemble Sepsis nitens but can be distinguished by the following characters: (1) Medioventral tubercle on male fore femur of $S$. spura is spur-like and bent at a forward angle with two smaller adjacent spines dorsally positioned at the end of the tubercle and one larger spine at the ventral end (Figs 24, 26), while the tubercle in $S$. nitens is thicker on the base and has its three spines positioned more in a anterio-posterior fashion (Fig. 21). (2) The basal laminalike projection on the fore tibia of $S$. spura (Figs 25, 27) merges back with the tibia gently, but ends off with a distinct lobe in S. nitens (Fig. 22). The short spines found posteriorly on the projection are also much weaker than those found in S. nitens. (3) Sepsis spura (Figs 25, 27) has only one anterior lamina-like protrusion on the distal portion of the fore tibia, while $S$. nitens (Fig. 22) has such protrusions on both anterior and posterior sides. (4) The surstylus of S. nitens (Fig. 23) has a rather angular basal swelling and is relatively straight, curved only at the terminus, while the surstyli of $S$. spura (Figs 30, 31) has a rounded basal swelling and is medially curved for the entirety of the surstylus. Sepsis spura can be distinguished from other Sepsis, also based on the specific structure of the male fore leg ornamentation and the shape of the surstylus.

Description (male). Colour. Head capsule mostly brown with a thin light brown strip on gena; fascial margin black. Vertex dark brown. Facial carina and lunule light grey-brown. Pedicel dark brown, $1^{\text {st }}$ flagellomere yellowish, arista brown. Proboscis whit- 


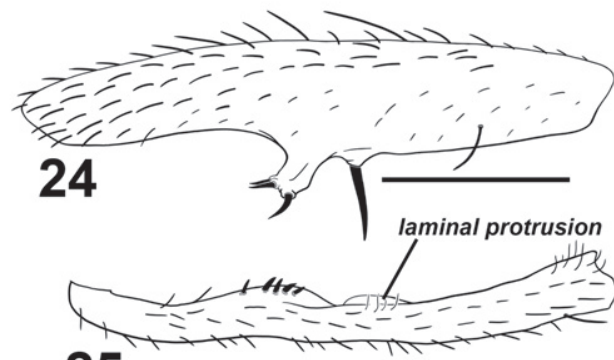

25

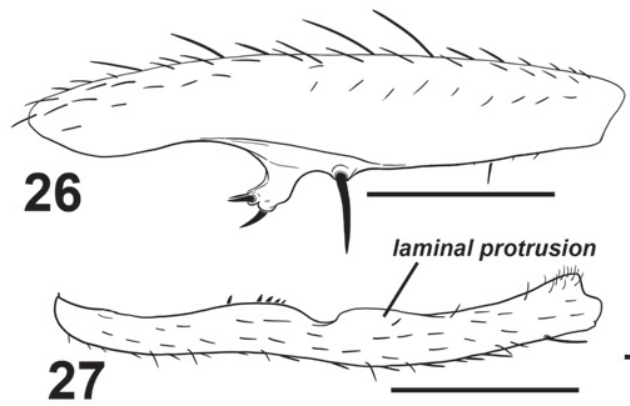

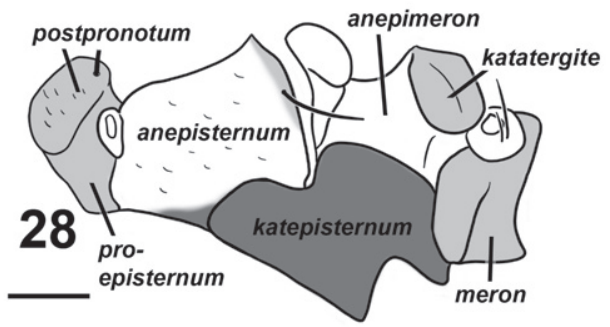

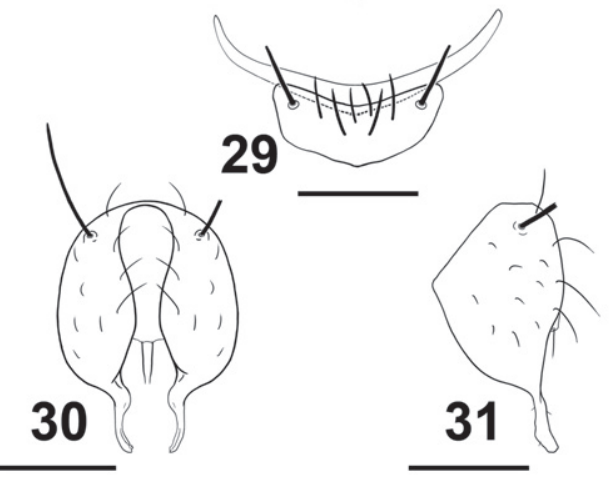

Figures 24-31. Sepsis spura. 24 fore femur, posterior $\mathbf{2 5}$ fore tibia, posterior $\mathbf{2 6}$ fore femur, anterior $\mathbf{2 7}$ fore tibia, anterior $\mathbf{2 8}$ pleural pruinosity pattern, lateral $\mathbf{2 9} 4^{\text {th }}$ and $5^{\text {th }}$ sternites, ventral $\mathbf{3 0}$ hypopygium, dorsal $\mathbf{3}$ I hypopygium, lateral. Scale bars: $0.5 \mathrm{~mm}$.

ish yellow. Forelegs wholly yellow. Mid and rear coxa yellow with brown base. Mid femur brown but yellow on basal and distal tips; mid tibia brown on basal half and diffuses to yellow on apical half. Rear femur yellow but brown on dorsal region, while rear tibia wholly brown. Fore tarsus with tarsomeres 3-5 brown, mid tarsus with tarsomeres 3-5 very lightly brown, rear tarsus with tarsomeres 4 and 5 brown. Wing clear except for basicostal cell and basal region of costal cell, which is light brown. Veins dark brown. Calypter clear, margin and fringe-hairs yellowish. Haltere white. Thorax mostly dark brown, but pronotopleuron is yellow. Abdominal tergites and sternites glossy dark brown.

Head. Roundish, facial carina short and shallow, facial area receding. Gena and parafacial region narrow. Largely glossy except for lightly microtomentose occipital region. Chaetotaxy: 1 ocellar, 1 divergent postocellar (ocellar longer than postocellar), 1 inner vertical, 1 outer vertical (outer subequal to inner). Orbital very reduced to absent. 3-4 vibrissae. 3-4 postocular. Lower fascial margin lined with setulae.

Thorax. Scutum, postpronotum and scutellum wholly microtomentose. Subscutellum microtomentose with a small glossy spot ventromedially. Mediotergite microtomentose on margins and glossy in the medial region. Scutellum twice wide as long. Pleural pruinosity pattern (Fig. 28): Proepisternum lightly microtomentose. Anepisternum largely glossy with a small strip on the anterioventral and posteriodorsal margins very lightly dusted. Balsare glossy. Katepisternum densely microtomentose. 
Anepimeron glossy. Katatergite, meron and metepimeron microtomentose. Chaetotaxy: 1 apical scutellar, 1 reduced basal scutellar, 1 row dorsocentral with posterior-most two setae as bristles, 1 row acrostichial and 1 postalar, 1 supraalar, 1 notopleural, 1 postpronotal, 1 anepisternal and 2 posterior spiracular.

Legs. Forelegs modified; fore femur (Figs 24, 26) with large submedian ventral spine and robust, forward curving spur-like protrusion at the median. This protrusion terminates with three short stout spines, with two adjacently positioned dorsally and one positioned ventrally. Fore tibia (Figs 25, 27) with a submedial and medial cuticular laminar extension; submedial extension with row of short spines. Additional chaetotaxy: Fore tibia with apical anteriodorsal. Mid femur with 1 anterior. Mid tibia with 2 posterior on median and subapex, 2 dorsal on basal 2/3 and apex, 1 anterior on apex, 1 anterioventral on basal 2/3 and 1 ventral on apex. Hind femur with 1 anteriodorsal on basal 2/3 and 1 posterioventral subapically. Hind tibia with 2 dorsal at median and subapically, 1 anterior at median and 2 anterioventral medially and subapically. Rear basitarsus with 2 ventral dark spines basally.

Wings. Without pterostigma. Veins bare. Covered with microtrichiae except for basal half of basal-medial cell. Anterior region of $\mathrm{r} 2+3$ cell with sparse microtrichiae. Radial-medial cross-vein divides discal-medial cell by ratio of slightly less than $2: 1$. Length: $2.9-3.5 \mathrm{~mm}$.

Abdomen. Tergites glossy black, syntergite $1+2-$ tergite 5 normal, tergite 6 missing, syntergite $7+8$ present and extending ventrad as a narrow sclerite. Spiracles $1-3$ on intersegmental membrane close to tergite, spiracles 4 and 5 within respective tergites near margin. Spiracles 7 and 8 adjacent on margin of syntergite $7+8$. Sternite 1 a broad rectangle with posterior invaginations while sternite 2 is triangular, tapering posteriorly; sternite 3 is oblong. Sternite 4 is V-shaped with setae and one stout discal bristle on each lateral margin; sternite 5 reduced to a thin lateral crescent (Fig. 29).

Hypopygium. Cercal plate with two very weak lobes, each with single distal setae. Hypopygium and surstylus relatively setaeless. Surstylus fused to hypopygium; bulging medially at base but thin and curved medially, slightly dentate terminally (Figs 30, 31).

Distribution. Indonesia (Sulawesi, Sumatra), Vietnam (Lào Cai).

\section{Key to species of the genus Perochaeta Duda, 1926 (males)}

1 Ratio of radial-medial cross-vein dividing discal-medial cell is $1: 1 \ldots \ldots \ldots \ldots \ldots . . .2$

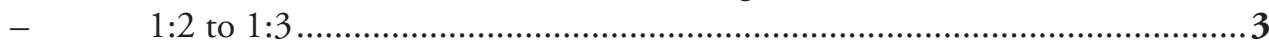

2 Surstylus without large medial protrusions; with small dentate terminus ...... Perochaeta orientalis Duda, 1926

- $\quad$ Sternite brush embedded in membrane....................................................4

$3 \quad$ Sternite brush as a free sclerotized lobe.................................................

- Sternite brush with few bristles (ca. 5 short and 5 long bristles); surstylus with large, squat triangular protrusion on inward median 

Sternite brush with few bristles (ca. 5 short bristles and 5 long bristles); surstylus without large median protrusions .....Perochaeta dikowi Ang et al., 2008

- $\quad$ Medial protrusion emerges dorsally on surstylus; posterior margin of $4^{\text {th }}$ sternite $\mathrm{V}$-shaped with many bristles Perochaeta cuirassa Ang, 2010 Medial protrusion emerges ventrally on surstylus; posterior margin of $4^{\text {th }}$ sternite as two distinct lobes with many bristles

Perochaeta lobo Ang, 2010

\section{Discussion}

\section{Notes on Perochaeta}

Perochaeta cuirassa and $P$. lobo are the first Perochaeta species from Vietnam. That they are found in the same locality is surprising, given that Perochaeta is a small Oriental genus with only three described species. As discussed in Ang et al. (2008), the species in this genus appear to be only found in mid- and high-elevation localities in Asia above 650m ASL: Perochaeta dikowi was found on Fraser's Hill, Malaysia (1300m ASL; Ang et al. 2008), P. hennigi is only known from Thawalamtenne, Sri Lanka (670m ASL; Ozerov 1992) and Tamil Nadu, India (1200m and $1400 \mathrm{~m}$ ASL; Iwasa and Tewari 1994). The type locality of $P$. orientalis is the Jiji Township in Taiwan ("Chip-chip" as designated by de Meijere; Duda 1926) which is within the Chung Yang mountain range that has an average elevation $>1000 \mathrm{~m}$ ASL, and can also found in regions such as Indonesia (Seram Is., $750 \mathrm{~m}$; Iwasa 2001). The new species $P$. cuirassa and $P$. lobo were found at $2600 \mathrm{~m}$ ASL. A male Perochaeta specimen from Flores Island, Indonesia, examined by Hennig (1941) was also collected from a mid elevation site (1200m ASL). Unfortunately, the latter was poorly preserved and could not be described, but based on Hennig's illustration, it likely constitutes a new species. Given that Perochaeta species are restricted to elevated sites, we predict that the number of species will rapidly grow as more mid and high elevation localities are sampled.

Adding two new species to Perochaeta is also of interest, because this (small) genus is very atypical for sepsids. Males of most sepsids have modified forelegs (e.g., cuticular tubercles and stout, enlarged spines; see Figs 15-16, 18-19, 21-22, 24-27) which are used to grab the base of female wings during mating (Pont and Meier 2002, Ingram et al. 2008, Puniamoorthy et al. 2008, 2009). However, Perochaeta has secondarily reduced foreleg armature, and at least $P$. dikowi has evolved a novel mounting behavior that does not involve the foreleg grasp (Ang et al. 2008). This has made Perochaeta a model for testing the correlation between the evolution of behavior and morphology (see Puniamoorthy et al. 2008, 2009). 


\section{Notes on Sepsis}

Sepsis spura appears to be a relatively widespread species ranging between Indonesia and Vietnam, and it can be found across all elevations, ranging from highlands (Sa Pa Valley, Vietnam; 1250m ASL) through mid elevation areas (Kampung Jawa, Indonesia; $650 \mathrm{~m} \mathrm{ASL}$ ) to sea level (Bandar Gadang Beach, Indonesia). Such widespread species of insects are relatively common in Southeast Asia and are increasingly attracting attention because they can give rise to new species (see Balke et al. 2009). At all localities the S. spura is found in low numbers on bovine dung while other Sepsis species can be very abundant. This, along with its morphological resemblance to $S$. nitens, may explain why $S$. spura is only described now. It is likely to belong to a clade of Sepsis species without wingspots (see Su et al. 2008).

Sepsis sepsi was first described from Sumbawa Is., Indonesia at 450m ASL (Ozerov 2003), but has subsequently been collected at low elevation sites in Sumatra as well as now at mid-elevation sites (800m ASL) in Ba Vi, Vietnam. This suggests that Sepsis sepsi is a relatively widespread species able to live in low to mid elevation habitats. We predict that it is also likely to be found in other areas between Indonesia and Vietnam. The record of the widely distributed Sepsis monostigma in Vietnam is not unexpected given that it falls within the recorded range from India to the far east of Russia.

\section{Acknowledgements}

The authors would like to thank Dr. Tran AD of Hanoi University of Science for the kind arrangement of the collection trip in North Vietnam, Dr. Iwasa M for his input on S. spura, Annie Ang for DNA sequence data on P. cuirassa and P. lobo, and Rachel Lin for translating Russian texts of the descriptions for P. hennigi and S. sepsi. This study was funded by the Singapore Ministry of Education grant R377-000-040-112.

\section{References}

Ang Y, Puniamoorthy N, Meier R (2008) Secondarily reduced foreleg armature in Perochaeta dikowi sp.n. (Diptera : Cyclorrhapha : Sepsidae) due to a novel mounting technique. Systematic Entomology 33: 552-559.

Balke M, Ribera I, Miller MA, Sagata K, Posman A, Vogler AP, Meier R (2009) New Guinea highland origin of a widespread arthropod supertramp. Proceedings of the Royal Society London 276: 2359-2367.

Brunetti E (1910) New Oriental Sepsinae. Records of the Indian Museum 3: 343-372.

Duda O (1926) Monographie der Sepsiden (Dipt.), II. Annalen des Naturhistorischen Museums in Wien 40: 1-110. 
Hennig W (1941) Dipteren von den kleinen Sunda-Inseln. Aus der Ausbeute der Sunda-Expedition Rensch. III. Piophilidae und Sepsidae. In Arbeiten über morphologische und taxonomische Entomologie aus Berlin-Dahlem 8: 145-149.

Ingram KK, Laamanen T, Puniamoorthy N, Meier R (2008) Lack of morphological coevolution between male forelegs and female wings in Themira (Sepsidae: Diptera: Insecta). Biological Journal of the Linnean Society 93: 227-238.

Iwasa M, Tewari RR (1994) Notes on the Sepsidae (Diptera) from India, with description of a new species. In: Shinonaga $S$ et al. (Eds) Studies on the taxonomy, ecology and control of the medically important flies in India and Nepal. Japanese Journal of Sanitary Zoology 45(Suppl.): 23-29.

Iwasa M (2001) Taxonomic and faunistic notes on the Sepsidae (Diptera) in Seram, Indonesia. Entomological Science 4: 533-538.

Iwasa M, Thinh TH (2008) Six new species of the genus Dicranosepsis Duda (Diptera, Sepsidae) from Vietnam, with a revised key to the species. Entomological Science 11: 65-73.

Laamanen TR, Petersen JFT, Meier R (2003) Kelp Flies and Species Concepts - The Case of Coelopa frigida and C. nebularum. Journal of Zoological Systematics and Evolutionary Research 41: 127-136.

Lim GS, Balke M, Meier R (In press) Determining species boundaries in a world full of rarity: singletons and species delimitation methods. Systematic Biology.

Meier R, Willmann R (2000) The Hennigian species concept. In Wheeler, QD, Meier, R (Eds) Species Concepts and Phylogenetic Theory: a Debate. Columbia University Press, New York, 30-43.

Merz B, Haenni J-P (2000) Morphology and terminology of adult Diptera (excluding terminalia). In Papp L, Darvas B (Eds) Contributions to a Manual of Palaearctic Diptera 1: General and Applied Dipterology. Science Herald, Budapest, 21-52.

Mishler B, Theriot E (2000) The phylogenetic species concept sensu Mishler and Theriot: monophyly, apomorphy, and phylogenetic species concepts. In Wheeler QD, Meier R (Eds) Species Concepts and Phylogenetic Theory: A Debate. Columbia University Press, New York, 44-54.

Ozerov AL (1992) On the taxonomy of flies of the family Sepsidae (Diptera). Byulleten' Moskovskogo obshchestva ispytateley prirody, Otd. Biol. 97(4): 44-47 [In Russian].

Overov AL (1993) A New Genus and Three New Species of Sepsidae (Diptera) from Vietnam. Entomol. Rev. 71(6): 123-127.

Ozerov AL (2003) On three species of the genus Sepsis Fallén (Diptera, Sepsidae). Zoologicheskiy zhurnal. 82(10): 1276-1280 [In Russian].

Ozerov AL (2005) World catalogue of the family Sepsidae (Insecta: Diptera). Zoologicheskie Issledovania 8, $74 \mathrm{pp}$.

Pont AC, Meier R (2002) The Sepsidae (Diptera) of Europe. Brill, 188 pp.

Puniamoorthy N, Su KF-Y, Meier R (2008) Bending for love: losses and gains of sexual dimorphisms are strictly correlated with changes in the mounting position of sepsid flies (Sepsidae: Diptera). BMC Evol Biol. 8:155. doi: 10.1186/1471-2148-8-155.

Puniamoorthy N, Ismail MR, Tan DSH, Meier R (2009) From kissing to belly stridulation: comparative analysis reveals surprising diversity, rapid evolution, and much homoplasy in 
the mating behaviour of 27 species of sepsid flies (Diptera: Sepsidae). Journal of Evolutionary Biology 22(11): 2146-56.

Sinclair BJ (2000) Morphology and terminology of Diptera male genitalia. In Papp L, Darvas B (Eds) Contributions to a Manual of Palaearctic Diptera 1: General and Applied Dipterology. Science Herald, Budapest, 53-74.

Su KF-Y, Kutty SN, Meier R (2008) Morphology versus Molecules: The phylogenetic relationships of Sepsidae (Diptera: Cyclorrhapha) based on morphology and DNA sequence data from ten genes. Cladistics 24: 902-916.

Tan DSH, Ali F, Kutty SN, Meier R (2008) The need for specifying species concepts: how many species of silvered langurs (Trachypithecus cristatus group) should be recognized? Molecular Phylogenetics and Evolution 49: 688-689.

Tan DSH, Ang Y, Lim GS, Ismail MR, Meier R (2010) From 'cryptic species' to integrative taxonomy: an iterative process involving DNA sequences, morphology, and behaviour leads to the resurrection of Sepsis pyrrhosoma (Sepsidae: Diptera). Zoologica Scripta 39: 51-61.

Thomson CG (1869) 6. Diptera. Species nova descripsit. In: Kongliga svenska fregatten Eugenies resa omkring Jorden. Stockholm, 2(1): 443-614.

Wheeler QD, Meier R (Eds) (2000) Species Concepts and Phylogenetic Theory: a Debate. Columbia University Press, New York, 230 pp. 